\title{
Could Planet Nine Be a Black Hole?
}

\author{
While astronomers look for a ninth planet to explain orbits in the outer \\ solar system, a duo explores the possibility that the mystery object is a \\ primordial black hole.
}

\section{By Christopher Crockett}

A peculiar alignment of orbits among some small bodies in the outer Solar System has astronomers hunting for a planet lurking well past Neptune. But what if this alleged Planet Nine-likely several times as massive as Earth and hundreds of times farther from the Sun-is not a planet but, instead, a diminutive black hole? This scenario is not only plausible, say Jakub Scholtz of Durham University, UK, and James Unwin of the University of Illinois at Chicago, but it may also help explain hints of small, dark, enigmatic objects flitting about our Galaxy.

The observation that the researchers seek to explain is an excess of free-floating interstellar objects spotted by the Optical Gravitational Lensing Experiment (OGLE) survey, which looks for starlight briefly magnified by the gravity of transiting bodies. Scholtz and Unwin point out that these objects, whose masses coincide with the range proposed for Planet Nine, could be primordial black holes formed from the collapse of overdense

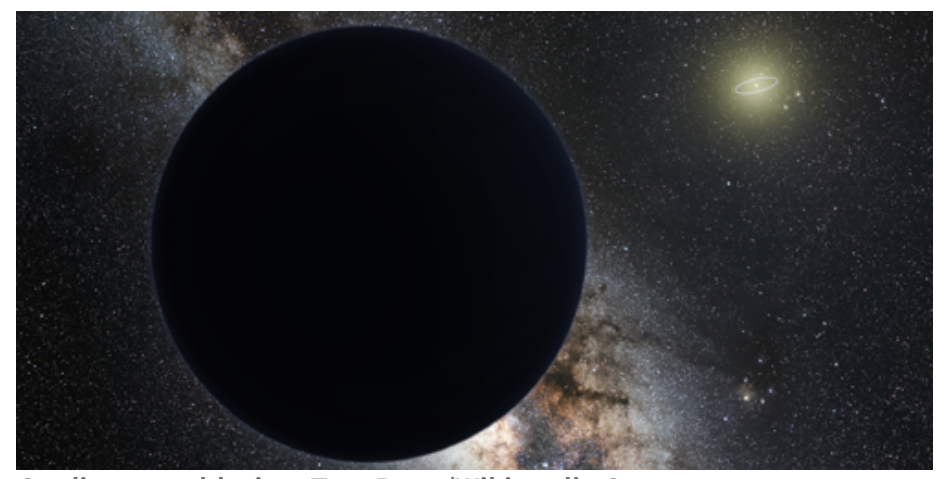

Credit: nagualdesign; Tom Ruen/Wikimedia Commons regions in the early Universe. They calculate that while the odds of the Sun capturing a wandering black hole are low, they're comparable to the probability of snagging a free-roaming planet (a common origin story for Planet Nine).

If ongoing searches for Planet Nine in visible and infrared light turn up empty, the pair says, then perhaps future surveys could look for a source of $x$ rays or gamma rays instead. Such a signal could be produced by particle annihilations within the dense dark matter that primordial black holes are thought to accumulate.

This research is published in Physical Review Letters.

Christopher Crockett is a freelance writer based in Arlington, Virginia. 